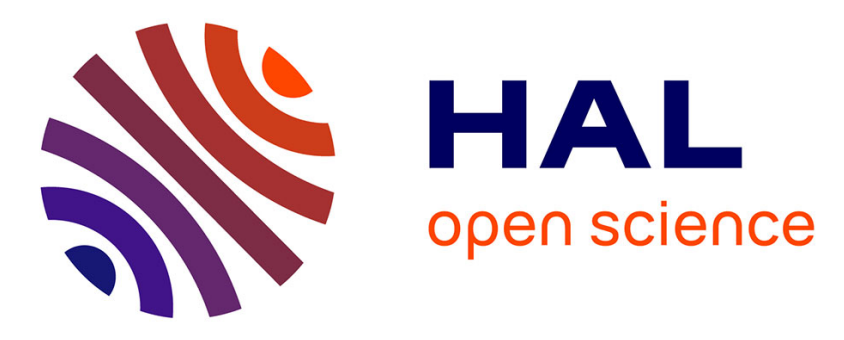

\title{
Modélisation des couplages fluide/solide dans les procédés d'assemblage à haute température
}

Thomas Heuzé, Jean-Baptiste Leblond, Jean-Michel Bergheau

\section{To cite this version:}

Thomas Heuzé, Jean-Baptiste Leblond, Jean-Michel Bergheau. Modélisation des couplages fluide/solide dans les procédés d'assemblage à haute température. Mécanique et Industries, 2011, 10.1051/meca/2011113 . hal-02279737

\section{HAL Id: hal-02279737 \\ https://hal.science/hal-02279737}

Submitted on 29 Nov 2019

HAL is a multi-disciplinary open access archive for the deposit and dissemination of scientific research documents, whether they are published or not. The documents may come from teaching and research institutions in France or abroad, or from public or private research centers.
L'archive ouverte pluridisciplinaire HAL, est destinée au dépôt et à la diffusion de documents scientifiques de niveau recherche, publiés ou non, émanant des établissements d'enseignement et de recherche français ou étrangers, des laboratoires publics ou privés.

\section{(c)(1)}

Distributed under a Creative Commons Attribution| 4.0 International License 


\title{
Modélisation des couplages fluide/solide dans les procédés d'assemblage à haute température
}

\author{
T. Heuzé, J.B. Leblond \\ UPMC, Institut Jean Le Rond d'Alembert, 4 place Jussieu tour 55-65, 75252 PARIS Cedex 05 \\ J.M. Bergheau \\ ENISE, LTDS, 58 rue Jean Parot 42023 Saint-Étienne Cedex 2
}

\begin{abstract}
Résumé On développe dans ces travaux un outil numérique permettant de simuler le procédé Friction Stir Spot Welding. Le modèle est basé sur un couplage fluide/solide permettant de décrire l'état de la matière dans toute la structure. Ce modèle s'appuie sur l'élément fini $\mathrm{P} 1+/ \mathrm{P} 1$, développé dans le cas d'un couplage thermomécanique fort, à la fois en mécanique des fluides et en mécanique des solides. Le couplage est associé avec une approche euléro-lagrangienne et intégré au sein d'une nouvelle option du code SYSWELD ${ }^{\circledR}$. On présente ici une première simulation du procédé Friction Stir Spot Welding.
\end{abstract}

Mots-clés Élément P1+/P1; Friction Stir Spot Welding; Couplage fluide/solide

\section{Introduction}

Le procédé de soudage ponctuel par friction et malaxage (Friction Stir Spot Welding [8]) est un procédé dérivé du soudage par friction et malaxage (Friction Stir Welding [10]) consistant à créer un joint de soudure «ponctuel » entre deux tôles superposées par pénétration d'un outil en rotation dans la matière. L'échauffement généré par le frottement ainsi que le mouvement de la matière entraînée génère deux phases, la première au voisinage de l'outil atteignant un état pâteux, l'autre restant solide.

Une approche eulérienne lagrangienne arbitraire (ALE), dans laquelle le maillage suit la matière dans la phase solide mais pas dans la zone pâteuse, permet de simuler de nombreux tours de l'outil dans la matière tout en suivant les bords des tôles. De plus, des modèles de comportement cohérents avec l'état de la matière dans chacune des zones peuvent être inclus, aussi la partie pâteuse est modélisée comme un fluide visqueux, et la partie solide avec une loi incluant les effets d'histoire.

Le modèle numérique développé est basé sur l'élément fini $\mathrm{P} 1+/ \mathrm{P} 1$ [1]. Cet élément mixte est développé avec une formulation température/vitesse/pression en mécanique des fluides (pour un écoulement laminaire incompressible transitoire $[3,4]$ ) et en mécanique des solides dans le cadre des grandes transformations.

Ces deux éléments sont couplés au sein d'une nouvelle option créée dans le code SYSWELD ${ }^{\circledR}$ [9], et présentés dans la section 3. La transition fluide/solide est effectuée au moyen d'un test explicite sur une température moyenne par élément, l'interface passe alors par les faces des éléments du maillage. Une procédure d'actualisation de la géométrie associée à l'approche euléro-lagrangienne est effectuée à convergence. Une première simulation simplifiée du procédé Friction Stir Spot Welding est présentée en section 4.

\section{Le procédé Friction Stir Spot Welding}

Le Friction Stir Spot Welding a été introduit au début des années 2000 par le constructeur automobile Mazda [8, 7]. Ce procédé dérivé du soudage par friction et malaxage consiste à effectuer un joint de soudure «ponctuel » entre deux tôles superposées par pénétration d'un outil, composé d'un pion et d'un épaulement, en rotation dans la matière. L'outil pénétrant dans la matière, un échauffement est généré à la fois par frottement au contact outil/matière, mais aussi par dissipation plastique dans la zone malaxée. L'échauffement de la matière ainsi que le mouvement de la matière entraînée au voisinage de l'outil permettent un mélange assurant la solidarité des parties à joindre après refroidissement. La figure 1 résume les trois étapes de la génération du joint soudé.

Le Friction Stir Spot Welding s'est vite avéré être un procédé très attractif notamment pour l'industrie automobile, venant directement concurrencer le procédé de soudage par résistance par point, en présentant la capacité de souder des alliages d'aluminium et des aciers haute résistance et en n'exigeant pas un apport d'énergie conséquent pour être mis en œuvre.

\section{Couplage fluide/solide}

Du point de vue de la modélisation, il est possible d'identifier deux « phases » de la matière dans les pièces à souder au cours du procédé (Figure 2). La première au voisinage de l'outil, est fortement malaxée et dans un état pâteux. Bien qu'elle n'atteigne pas le point de fusion, les forts taux de déformation y régnant nous autorisent à considérer la matière comme un fluide très visqueux. La seconde, composant la structure de base, se déforme de façon classique comme un solide et présente des effets d'histoire. 


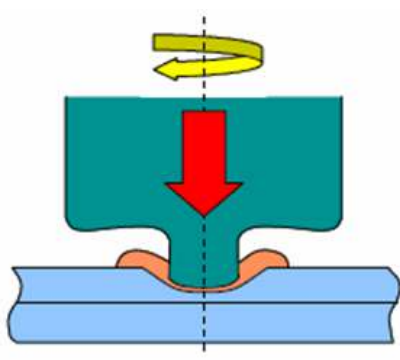

Plongée

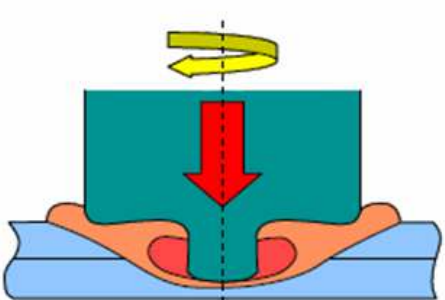

Malaxage

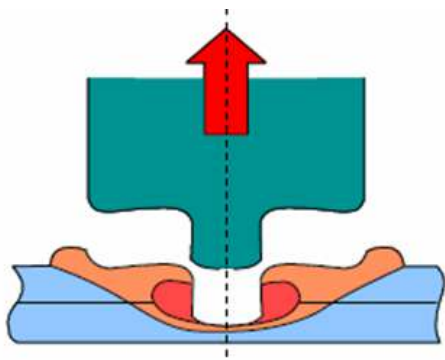

\section{Retrait}

Figure 1: Étapes du procédé Friction Stir Spot Welding

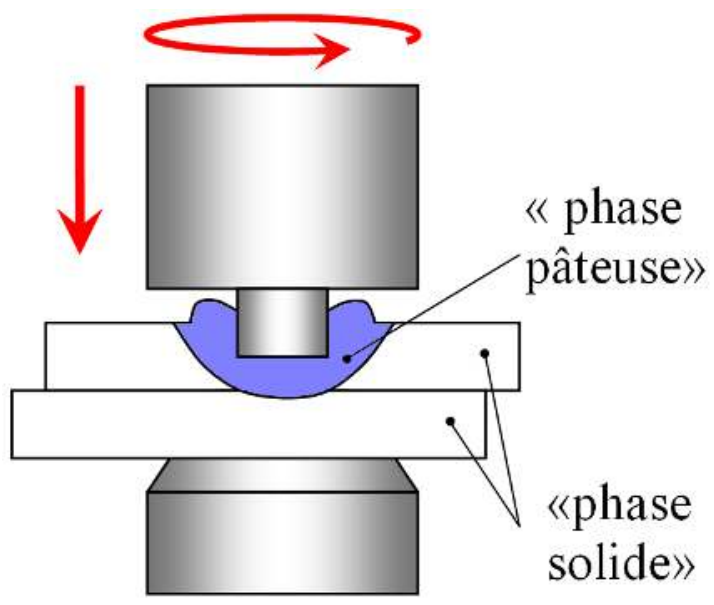

Figure 2: Phases identifiée lors du procédé Friction Stir Spot Welding

Pour modéliser numériquement ce procédé, les approches purement lagrangienne et eulérienne ne sont pas appropriées, l'une nécessitant de fréquents remaillages du fait des grandes déformations et l'autre ne permettant pas de suivre les bords des tôles pendant le procédé. Une approche eulérienne lagrangienne arbitraire (ALE), dans laquelle le maillage suit la matière dans la phase solide mais pas dans la zone pâteuse, peut permettre de simuler de nombreux tours de l'outil dans la matière tout en suivant les bords des tôles. En plus du traitement numérique différent des deux zones, des modèles de comportement cohérents avec l'état de la matière peuvent être utilisés dans chaque zone en y intégrant respectivement des descriptions de type fluide visqueux incompressible et solide. Le couplage fluide/solide alors construit présente cette particularité qu'au cours du procédé, une zone va progresser au détriment de l'autre par chauffage (et malaxage) ou refroidissement.

\subsection{Comportements thermomécaniques fluide et solide}

La partie pâteuse est modélisée comme un fluide visqueux incompressible et décrite avec une loi de Norton-Hoff. Le solide est modélisé en grandes transformations, on postule une partition additive du taux de déformation eulérien $\mathbf{D}$ faisant donc intervenir une loi d'hypoélasticité. Les équations (1) résument les équations constitutives associées aux comportements mécaniques fluide et solide :

\begin{tabular}{|l|l|}
\hline Fluide & Solide \\
\hline \multicolumn{2}{|c|}{$\boldsymbol{\sigma}=p \mathbf{1}+\mathbf{s}$} \\
$\mathbf{q}=-k \boldsymbol{\nabla} T$ \\
\hline $\mathbf{s}=2 \mu \mathbf{D}$ & $\mathbf{D}=\mathbf{D}^{e}+\mathbf{D}^{p}+\dot{\varepsilon}^{t h} \mathbf{1}$ \\
$\mu=K\left(\sqrt{3} D_{e q}\right)^{m-1}$ & $\mathbf{s}=\mathcal{F}(\mathbf{v}(\tau), \tau \leq t)$ \\
$D_{e q}=\sqrt{\frac{2}{3} \mathbf{D}: \mathbf{D}}$ & $\mathbf{D}^{e}=\frac{1+\nu}{E} \stackrel{\nabla}{\boldsymbol{\sigma}}-\frac{\nu}{E}(\operatorname{tr} \quad \stackrel{\nabla}{\boldsymbol{\sigma}}) \mathbf{1}$ \\
$\operatorname{div} \mathbf{v}=0$ & $\operatorname{tr} \mathbf{D}^{p}=0$ \\
\hline
\end{tabular}

où $\stackrel{\nabla}{\boldsymbol{\sigma}}$ est une dérivée objective des contraintes de Cauchy, $p$ l'opposé de la pression hydrostatique, $\mathbf{s}$ le déviateur des contraintes, q le flux surfacique de chaleur, $\mu$ la viscosité dynamique du fluide définie à partir des paramètres $K$ (consistance) et $m$ (sensibilité), $E$ et $\nu$ le module d'Young et le coefficient de Poisson, $k$ la conductivité thermique, $\dot{\varepsilon}^{t h}$ le taux de déformation «thermométallurgique » provenant des dilatations thermiques et des changements de volume associés à la métallurgie, et $\mathcal{F}$ est une fonctionnelle décrivant la relation de comportement dépendant de l'histoire du solide, pouvant dépendre ou non de la vitesse de chargement. Les paramètres matériau dépendent a priori de la température. 


\subsection{Forme faible du problème}

L'échauffement et le malaxage de la matière au cours du procédé Friction Stir Spot Welding nécessitent d'intégrer un couplage thermomécanique fort au modèle. Aussi le problème à résoudre fait intervenir les équations d'énergie, de mouvement et les liaisons internes de chaque zone. La formulation faible mixte du problème s'écrit sous la forme suivante, en notant $\chi$ les coordonnées associées à la configuration ALE :

$$
\begin{aligned}
& \text { Trouver } \left.\left.(T, \mathbf{v}, p) \in\left(\mathcal{T}_{a d} \times \mathcal{V}_{a d} \times \mathcal{P}_{a d}\right), \forall t \in\right] 0, T\right], \text { tel que } \forall\left(T^{*}, \mathbf{v}^{*}, p^{*}\right) \in\left(\mathcal{T}_{a d}^{0} \times \mathcal{V}_{a d}^{0} \times \mathcal{P}_{a d}\right), \\
& -\int_{\Omega_{\chi}} k \boldsymbol{\nabla} T \cdot \boldsymbol{\nabla} T^{*} \mathrm{~d} \Omega+\int_{\partial \Omega_{q}} \phi^{d} T^{*} \mathrm{~d} S+\int_{\Omega_{\chi}}\left\{\begin{array}{c}
\boldsymbol{\sigma}: \mathbf{D} \\
\beta \boldsymbol{\sigma}: \mathbf{D}^{p}
\end{array}\right\} T^{*} \mathrm{~d} \Omega=\int_{\Omega_{\chi}} \rho C\left(\left.\frac{\partial T}{\partial t}\right|_{\chi}+\left\{\begin{array}{c}
\boldsymbol{\nabla} T \cdot \mathbf{c} \\
0
\end{array}\right\}\right) T^{*} \mathrm{~d} \Omega \\
& -\int_{\Omega_{\chi}}\left\{\begin{array}{c}
2 \mu \mathbf{D} \\
\mathbf{s}(\mathbf{v}(\tau), \tau \leq t)
\end{array}\right\}: \mathbf{D}^{*} d \Omega-\int_{\Omega} p \operatorname{div} \mathbf{v}^{*} \mathrm{~d} \Omega+\int_{\partial \Omega_{F}} \mathbf{F}_{d} \cdot \mathbf{v}^{*} \mathrm{~d} S=\int_{\Omega_{\chi}} \rho\left(\left\{\begin{array}{c}
\left.\frac{\partial \mathbf{v}}{\partial t}\right|_{\chi}+\nabla \mathbf{v} \cdot \mathbf{c} \\
\mathbf{0}
\end{array}\right\}\right) \mathbf{v}^{*} \mathrm{~d} \Omega \\
& \int_{\Omega_{\chi}} p^{*}\left(\operatorname{div} \mathbf{v}-\left\{\begin{array}{c}
0 \\
\frac{\dot{p}}{\kappa}+3 \dot{\varepsilon}^{t h}
\end{array}\right\}\right) \mathrm{d} \Omega=0 \\
& T(\mathbf{x}, t=0)=T_{0} \\
& \mathbf{v}(\mathbf{x}, t=0)=\mathbf{v}_{0}
\end{aligned}
$$

où $\mathbf{c}=\mathbf{v}-\hat{\mathbf{v}}$ est la vitesse convective définie à partir de la vitesse matérielle $\mathbf{v}$ et de la vitesse du maillage $\hat{\mathbf{v}}, \phi^{d}$ et $\mathbf{F}_{d}$ représentent le flux surfacique de chaleur et les tractions surfaciques imposés, $\rho$ la masse volumique, $C$ la capacité calorifique, $\beta$ le coefficient de Quinney-Taylor généralement fixé à 0.9 et $\kappa$ le module de compressibilité élastique. Les accolades dans les équations permettent de distinguer les termes associés aux phases fluide (en haut) et solide (en bas), cette représentation symbolique sera conservée par la suite. On note que les termes d'inertie mécanique ont été négligés dans la partie solide, le processus de déformation pouvant y être considéré comme quasi-statique.

\subsection{L'élément fini $P 1+/ P 1$}

L'élément fini P1+/P1 ou élément MINI sert de base pour le développement du couplage. Initialement proposé par Arnold et al. [1], il s'inscrit dans le cadre des méthodes mixtes et permet le traitement de liaisons internes de type incompressibilité totale ou partielle. Cet élément linéaire enrichi s'appuie sur le tétraèdre à quatre nœuds (Figure 3), et assure dans la version proposée, la continuité des champs de température, vitesse et pression. Aussi cinq degrés de

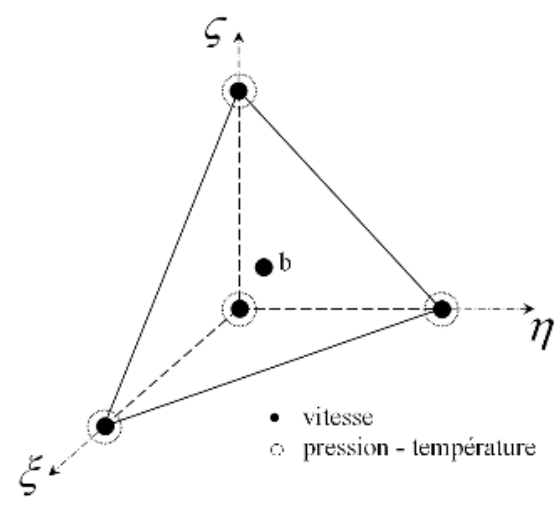

Figure 3: Tétraèdre $\mathrm{P} 1+/ \mathrm{P} 1$ de référence.

liberté sont associés à chaque nœud sommet du tétraèdre. L'approximation du champ de vitesse est enrichie en intégrant un nœud interne supplémentaire, positionné au barycentre de l'élément, auquel est associée une « fonction bulle » (approximation $\mathrm{P} 1+$ ). On rappelle que ce type de fonction s'annule sur le pourtour de l'élément. Cet enrichissement permet de satisfaire à la fois les équations du mouvement et la liaison interne considérée. On montre aussi que cet élément satisfait les conditions de Brezzi-Babuska.

Le champ de vitesse est interpolé sur l'élément comme suit :

$$
\mathbf{v}^{h}(\mathbf{x})=N^{(p)}(\xi, \eta, \zeta) \mathbf{v}^{(p)}+N^{(b)}(\xi, \eta, \zeta) \boldsymbol{\lambda} \quad 1 \leq p \leq 4
$$

où $p$ est le numéro d'un nœud sommet de l'élément, $b$ l'indice relatif au nœud bulle et $\boldsymbol{\lambda}$ est le vecteur des degrés de liberté liés au nœud bulle, homogènes à des vitesses.

\subsection{Formulation éléments finis}

La discrétisation par éléments finis de la forme faible (2) conduit à l'écriture du système non linéaire d'équations semi-discrètes suivant :

$$
\mathbf{M} \dot{\mathbf{q}}+\left\{\begin{array}{c}
\mathbf{f}^{\mathrm{conv}} \\
\mathbf{0}
\end{array}\right\}+\mathbf{f}^{\mathrm{int}}=\mathbf{f}^{\mathrm{ext}}
$$

où $\mathbf{q}$ est le vecteur des degrés de liberté du système, et s'écrit de façon symbolique $\mathbf{q}^{T}=\{\mathbf{T} \mathbf{v} \mathbf{p} \boldsymbol{\lambda}\}$. La matrice de masse généralisée $\mathbf{M}$ contient les termes d'inertie mécanique (seulement pour la partie fluide) et thermique. Les efforts 
internes $\mathbf{f}^{\text {int }}$, externes $\mathbf{f}^{\text {ext }}$, et les efforts dûs aux termes de convection $\mathbf{f}^{\text {conv }}$ sont assemblés à partir des quantités élémentaires et définis, en détaillant les composantes associées au $p^{\mathrm{e}}$ nœud sommet $(p=1$ à 4$)$ et au nœud bulle $(b)$, de la façon suivante :

$$
\begin{aligned}
& \mathbf{f}^{\text {int }}=\sum_{e=1}^{N_{e l}}\left(\left\{\begin{array}{l}
f_{T}^{\text {int, }(p)}=-\int_{\Omega_{e}} \nabla N^{(p)} \cdot \mathbf{q d} \Omega \\
\mathbf{f}_{v}^{\text {int, }(p)}=\int_{\Omega_{e}} \mathbf{B}_{p}^{T} \mathbf{s d} \Omega+\int_{\Omega_{e}} \boldsymbol{\nabla} N^{(p)} p \mathrm{~d} \Omega \\
\operatorname{div} \mathbf{v} \\
f_{p}^{\text {int, }(p)}=\int_{\Omega_{e}} N^{(p)}\left\{\begin{array}{c}
\operatorname{div} \mathbf{v}-\frac{\dot{p}}{\kappa}-3 \dot{\varepsilon}^{\text {th }}
\end{array}\right\} \mathrm{d} \Omega \\
\mathbf{f}_{b}^{\text {int }}=\int_{\Omega_{e}} \mathbf{B}_{b}^{T} \mathbf{s} \mathrm{d} \Omega+\int_{\Omega_{e}} \nabla N^{(b)} p \mathrm{~d} \Omega
\end{array}\right\}\right. \\
& \left.\mathbf{f}^{\text {ext }}=\sum_{e=1}^{N_{e l}}\left\{\begin{array}{l}
f_{T}^{\text {ext },(p)}=\int_{\partial \Omega_{e} \cap \partial \Omega_{q}} \phi^{d} N^{(p)} \mathrm{d} S+\int_{\Omega_{e}}\left\{\begin{array}{c}
\boldsymbol{\sigma}: \mathbf{D} \\
\beta \boldsymbol{\sigma}: \mathbf{D}^{p}
\end{array}\right\} N^{(p)} \mathrm{d} \Omega \\
\mathbf{f}_{v}^{\text {ext },(p)}=\int_{\partial \Omega_{e} \cap \partial \Omega_{F}} \mathbf{F}_{d} N^{(p)} \mathrm{d} S \\
0 \\
\mathbf{f}_{b}^{\text {ext }}=\mathbf{0}
\end{array}\right\}{ }^{(p)}\right) \\
& \left.\mathbf{f}^{\text {conv }}=\sum_{e=1}^{N_{e l}}\left(\begin{array}{l}
f_{T}^{\text {conv },(p)}=\int_{\Omega_{e}} \rho C(\nabla T) \cdot \mathbf{c} \bar{N}^{(p)} \mathrm{d} \Omega \\
\mathbf{f}_{v}^{\text {ext },(p)}=\int_{\Omega_{e}} \rho(\nabla \mathbf{v}) \cdot \mathbf{c} N^{(p)} \mathrm{d} \Omega \\
0 \\
\mathbf{f}_{b}^{\text {conv }}=\int_{\Omega_{e}} \rho(\nabla \mathbf{v}) \cdot \mathbf{c} N^{(b)} \mathrm{d} \Omega
\end{array}\right\}\right)
\end{aligned}
$$

où la notation $\sum_{e=1}^{N_{e l}}$ dénote l'opération d'assemblage des quantités élémentaires, $\mathbf{B}_{p}$ est la matrice contenant les gradients de la fonction de forme associée au noud $p$, reliant les taux de déformation aux composantes de la vitesse du nœud $p$. On rappelle que $\int \mathbf{F}_{d} N^{(b)} \mathrm{d} S=\mathbf{0}$ car la fonction bulle s'annule sur le pourtour de l'élément.

Un algorithme aux différences finies de type Euler implicite est utilisé pour la résolution. Dans le cas de la méthode de Newton-Raphson, la linéarisation des équations discrètes effectuée à l'itération $k$ du calcul conduit à la résolution du système matriciel suivant :

$$
\mathbf{M}^{(k)} \dot{\mathbf{q}}^{(k+1)}+\mathbf{K}^{(k)} \delta \mathbf{q}^{(k)}=\mathbf{R}^{(k)}
$$

où l'incrément $\delta \mathbf{q}^{(k)}$ est défini par $\delta \mathbf{q}^{(k)}=\mathbf{q}^{(k+1)}-\mathbf{q}^{(k)}$. Dans ce système, $\dot{\mathbf{q}}^{(k+1)}$ est lié à $\delta \mathbf{q}^{(k)}$ par le pas de temps $\Delta t$. La matrice de rigidité $\mathbf{K}^{(k)}$ et le vecteur résidu $\mathbf{R}^{(k)}$ sont définis comme suit :

$$
\mathbf{R}^{(k)}=\mathbf{f}^{\text {ext }}-\left\{\begin{array}{c}
\mathbf{f}^{\mathrm{conv}} \\
\mathbf{0}
\end{array}\right\}-\mathbf{f}^{\mathrm{int}}, \quad \mathbf{K}^{(k)}=-\left.\frac{\partial \mathbf{R}}{\partial \mathbf{q}}\right|^{(k)}
$$

On notera que $\mathbf{R}^{(k)}$ ne s'annule pas à la convergence des itérations. Cette présentation est cohérente avec la structure du code : la routine élémentaire calcule les matrices de masse et de rigidité et le vecteur résidu, et la discrétisation temporelle des termes d'inertie est effectuée par une autre routine dans la boucle sur les mailles. Le système linéaire (6) peut être détaillé de façon symbolique, conservant la distinction entre accolades des termes associés aux phases fluide (en haut) et solide (en bas), sous la forme suivante :

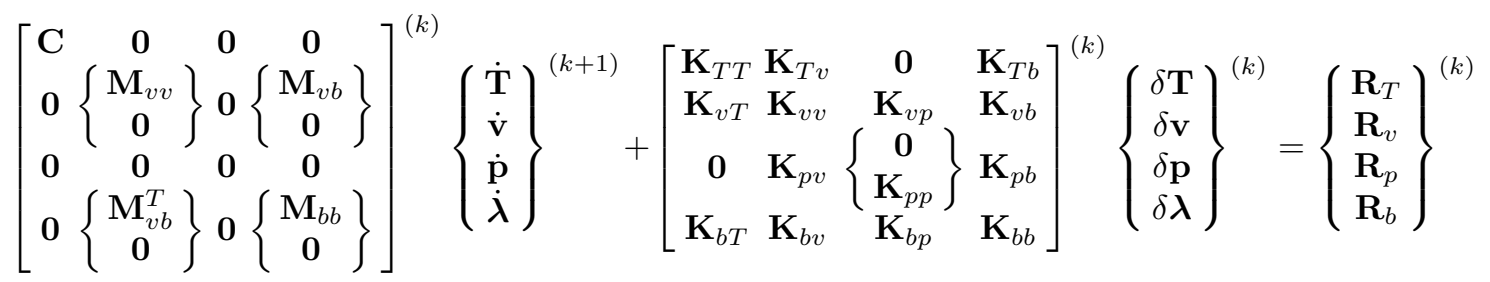

La matrice de rigidité du système (8) est assemblée à partir des contributions des sous-matrices définies à partir des degrés de liberté température, vitesse et pression des nœuds sommets et des degrés de liberté associés au nœud bulle :

$$
\mathbf{K}_{x y}=-\frac{\partial \mathbf{R}_{x}}{\partial \mathbf{y}}, \quad(\mathbf{x}, \mathbf{y})=(\mathbf{T}, \mathbf{v}, \mathbf{p}, \boldsymbol{\lambda})
$$

On note aussi que (i) les termes d'inertie mécanique sont omis dans la partie solide et que (ii) la sous-matrice $\mathbf{K}_{p p}$ intervenant dans la partie solide provient de l'élasticité présente dans son comportement mécanique, plus précisément au travers du module de compressibilité élastique $\kappa$ apparaissant dans la formulation faible (2).

Pour gagner du temps lors de la résolution, le nœud bulle est condensé élément par élément dans les équations relatives aux nœuds sommets. Dans la partie fluide, la condensation est effectuée en introduisant une approximation 
l'accélérération du champ bulle [4,5]. La résolution à chaque itération est alors effectuée sur l'incrément du vecteur des degrés de liberté $\overline{\mathbf{q}}$ réduit après condensation :

$$
\mathbf{A}^{(k)} \delta \overline{\mathbf{q}}^{(k)}=\mathbf{B}^{(k)} \quad \text { avec } \quad \mid \begin{aligned}
& \mathbf{A}^{(k)}=\left(\frac{\overline{\mathbf{M}}^{(k)}}{\Delta t}+\overline{\mathbf{K}}^{(k)}\right) \\
& \mathbf{B}^{(k)}=\mathbf{\mathbf { R }}^{(k)}-\overline{\mathbf{M}}^{(k)} \dot{\mathbf{q}}^{(k)}
\end{aligned}
$$

\subsection{Repérage de l'interface fluide/solide}

La définition d'un élément comme solide ou fluide conduit à faire passer l'interface fluide/solide par les faces entre les éléments. Au cours du calcul, un élément peut changer de comportement, le critère le plus simple à mettre en place porte sur une température moyenne de l'élément. Une température de transition de phase est spécifiée par l'utilisateur dans la mise en données du calcul. De plus, pour faciliter la convergence du calcul, le test est effectué de manière explicite. Le calcul du résidu à l'instant $t+\Delta t$ est donc fait sur la base d'un test effectué sur une température moyenne par élément calculée à l'instant $t$, aussi au cours des itérations du calcul la distribution d'éléments fluides et solides demeure identique dans la structure. On observe que les éléments étant tous deux formulés en température/vitesse/pression, les quantités élémentaires sont automatiquement compatibles et s'assemblent de façon classique pour former la matrice premier membre et le résidu globaux. On voit ici tout l'intérêt d'avoir formulé l'élément solide en vitesse.

\subsection{Actualisation de la géométrie}

Le mouvement arbitraire associé à l'approche euléro-lagrangienne privilégiée ici permet d'éviter de suivre le mouvement de rotation de l'outil, et donc le coût de calcul et les erreurs numériques associées aux remaillages dans le cas d'une approche purement lagrangienne. Il est intéressant de noter que les fluides présentent généralement un comportement instantané, aussi il n'est pas besoin d'effectuer de transports de quantités accompagnant la procédure d'actualisation de la géométrie associée à l'approche ALE. C'est ici un intérêt tout particulier d'avoir choisi de représenter la zone malaxée comme un fluide.

Dans l'approche ALE développée ici, la procédure d'actualisation de la position des nœuds du maillage de la partie fluide est effectuée à la convergence du calcul, elle se déroule en trois temps :

1. les nœuds sont codés en fonction de la zone dans laquelle ils se trouvent (Figure 4(a)),

2. la position des nœuds de la surface libre est actualisée à partir de la projection normale de la vitesse matérielle convergée (Figure $4(\mathrm{~b})) \hat{\mathbf{v}}^{(p)}=\left(\mathbf{v}^{(p)} \cdot \mathbf{n}^{(p)}\right) \mathbf{n}^{(p)}$ où $\hat{\mathbf{v}}^{(p)}$ représente la vitesse de maillage du nœud $p$. La normale $\mathbf{n}^{(p)}$ au nœud $p$ de la surface libre est définie à partir de la moyenne des normales des mailles de peau constituant cette surface libre :

$$
\mathbf{n}^{(p)}=\sum_{e=1}^{N_{\mathrm{mp}}} \mathbf{n}_{E_{e}} /\left\|\sum_{e=1}^{N_{\mathrm{mp}}} \mathbf{n}_{E_{e}}\right\|
$$

où $N_{\mathrm{mp}}$ dénote le nombre de mailles de peau contenant le nœud $p$.

3. enfin les nœuds intérieurs de la zone fluide sont replacés de la façon la plus régulière possible par une technique de replacement barycentrique (Figure 4(c)). Cette technique consiste à repositionner le nœud $p$ au barycentre de

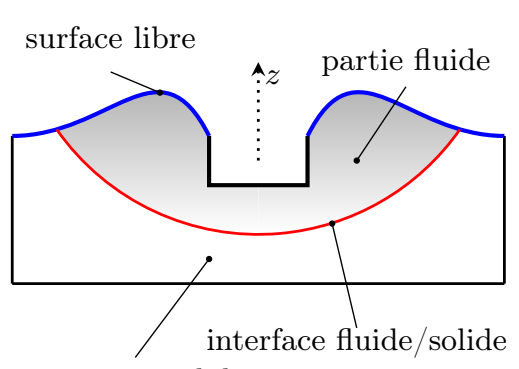
partie solide

(a) Codage des nœuds en zones

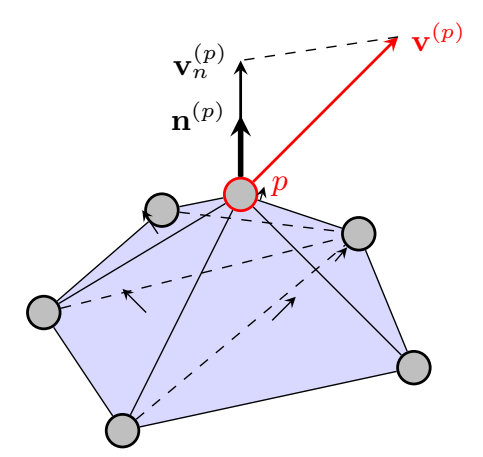

(b) Normale en un nœud

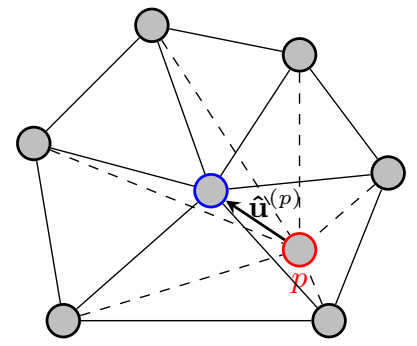

(c) Barycentrage

Figure 4: Actualisation de la géométrie

ses voisins :

$$
\mathbf{x}_{p}^{(\mathrm{it})}=\sum_{n=1}^{N_{\mathrm{vois}_{p}}} \mathbf{x}_{n}^{(\mathrm{it})} / N_{\text {vois }_{p}}
$$

où $N_{\text {vois }_{p}}$ représente le nombre de nœuds voisins du nœud $p$. Cette méthode est itérative et converge lorsque les nœuds sont tous placés au barycentre de leurs voisins. Le critère d'arrêt est posé sur un incrément de déplacement entre deux itérations $\sup _{i, p}\left(\delta \hat{u}_{i}^{(p)}\right)^{(\mathrm{it})}, i=1,3, p=1, N_{\max }$, qui doit être inférieur à une tolérance fixée. 


\section{Simulation numérique du Friction Stir Spot Welding}

On présente une simulation simplifiée du Friction Stir Spot Welding : (i) le modèle développé n'incluant pas pour l'instant le contact unilatéral, la simulation est menée sur une structure monobloc, on simule alors un essai d'enfoncement d'un pion en rotation dans la matière, (ii) le contact frottant est modélisé dans une première approche par des tractions surfaciques tangentielles réparties uniformément en fonction du rayon et un flux de chaleur uniforme sur la surface de contact.

\subsection{Mise en données}

La structure maillée (Figure 5(a)) possède un rayon de $35 \mathrm{~mm}$ et une épaisseur de $4 \mathrm{~mm}$. Le problème compte 29105 degrés de liberté. Les conditions aux limites thermiques et mécaniques sont détaillées sur les figures 5(b) et 5(c) respectivement pour les faces supérieure et inférieure de la structure. Un flux de chaleur constant de $6 \mathrm{~W} / \mathrm{mm}^{2}$ et

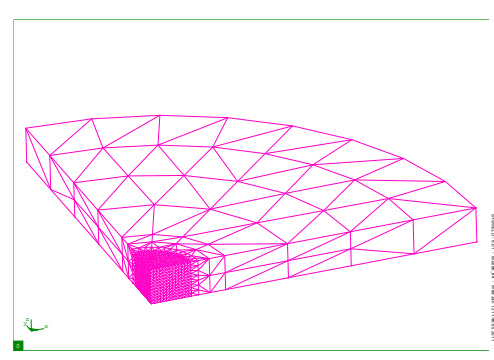

(a) Quart du maillage

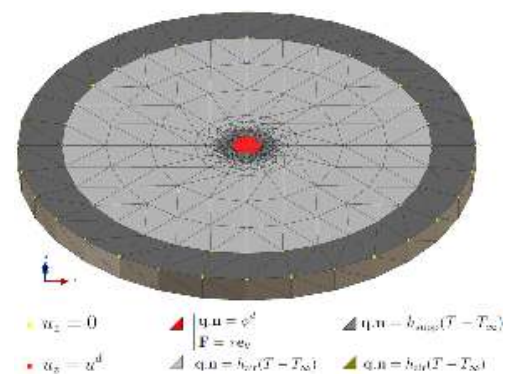

(b) Face supérieure

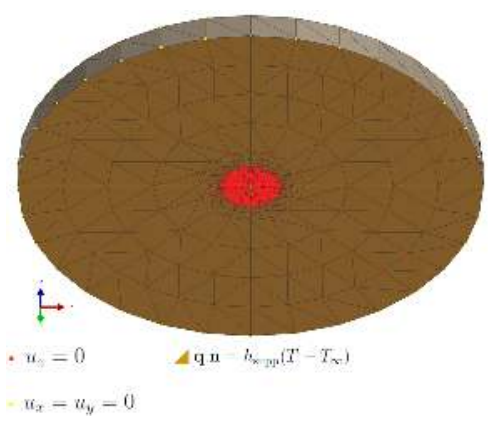

(c) Face inférieure

FIGURE 5: Maillage et conditions aux limites

des tractions surfaciques tangentielles de 600 MPa sont imposés au niveau de la zone de frottement. La température de transition fluide/solide est fixée de façon arbitraire à $100^{\circ} \mathrm{C}$ et la température extérieure est prescrite à $20^{\circ} \mathrm{C}$. Les valeurs des paramètres matériau correspondent à un alliage d'aluminium 2024 et sont regroupées dans le tableau 1. La figure 6(a) présente sous forme unidimensionnelle la relation élastoplastique utilisée, et les figures 6(b) et 6(c) les

\begin{tabular}{|l|l|l|l|}
\hline Module d'Young $E$ & $73000 \mathrm{MPa}$ & Conductivité thermique $k$ & $121 \mathrm{~W} /(\mathrm{m} . \mathrm{K})$ \\
\hline Coefficient de Poisson $\nu$ & 0.34 & Masse volumique $\rho$ & $2770 \mathrm{~kg} / \mathrm{m}^{3}$ \\
\hline Limite d'élasticité $\sigma_{y}$ & $350 \mathrm{MPa}$ & Capacité calorifique $C$ & $875 \mathrm{~J} /(\mathrm{kg} . \mathrm{K})$ \\
\hline Module d'écrouissage $h$ & $12000 \mathrm{MPa}$ & Coefficient échange air $h_{\text {air }}$ & $10 \mathrm{~W} /\left(\mathrm{m}^{2} . \mathrm{K}\right)$ \\
\hline Consistance (loi fluide) $K$ & $10 \mathrm{MPa} . \mathrm{s}$ & Coefficient échange support $h_{\text {supp }}$ & $350 \mathrm{~W} /\left(\mathrm{m}^{2} . \mathrm{K}\right)$ \\
\hline Sensibilité (loi fluide) $n$ & 1 & & \\
\hline
\end{tabular}

TABLE 1: Paramètres matériau

variations de la limite d'élasticité et de la consistance du fluide avec la température.

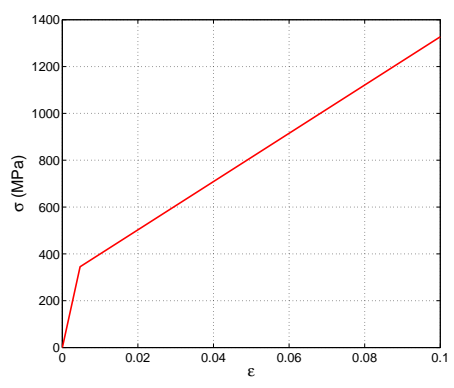

(a) Courbe de traction

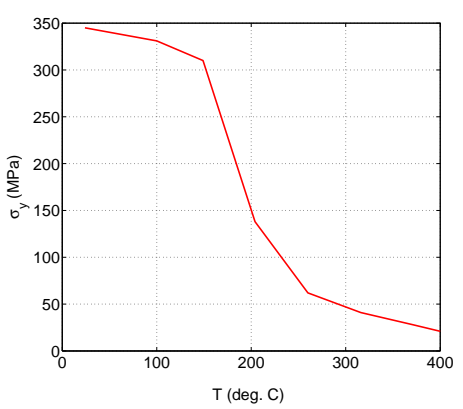

(b) Limite d'élasticité en traction

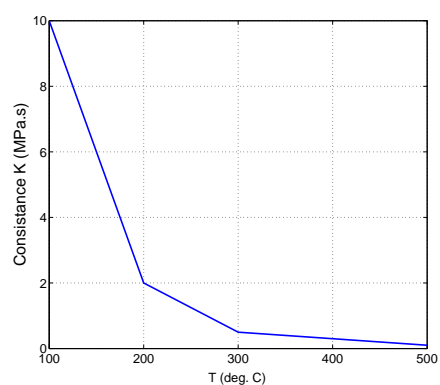

(c) Consistance du fluide

FiguRE 6: Relation élastoplastique 1D et paramètres matériau variant avec la température.

\subsection{Résultats}

On présente sur la figure 7 l'évolution du champ de température pendant la première partie du calcul. On observe bien l'apparition d'une zone chaude sous l'outil, qui commence d'abord à se propager sous la forme d'une coupole hémisphérique puis de façon radiale sur la structure. En concordance avec le champ de température, la zone fluide apparaît peu après le début de la simulation au voisinage de la zone de contact outil/matière et progresse de façon hémisphérique au cours du temps. Cette progression est illustrée au travers de l'évolution de l'isotherme associée à 
l'interface fluide/solide sur la figure 8. La figure 9 présente le champ de vitesse, on observe bien la transition solide/fluide entre les figures 9(a) et 9(b). Le champ est tout d'abord réparti. Dès qu'une zone fluidifie, toute la déformation s'y concentre, on assiste alors à un retour élastique de la partie solide environnante. Durant le procédé, l'outil créée des contraintes dans la zone où il appuie (Figure 10), mais ces contraintes chutent quand le solide se transforme en fluide. Les contraintes sont donc maximales dans la partie solide au voisinage de l'interface fluide/solide.

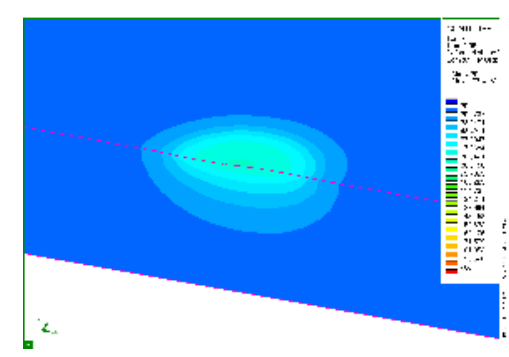

(a) $t=0.05 \mathrm{~s}$

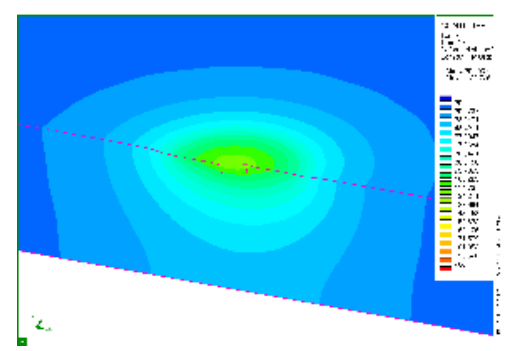

(b) $t=0.4 \mathrm{~s}$

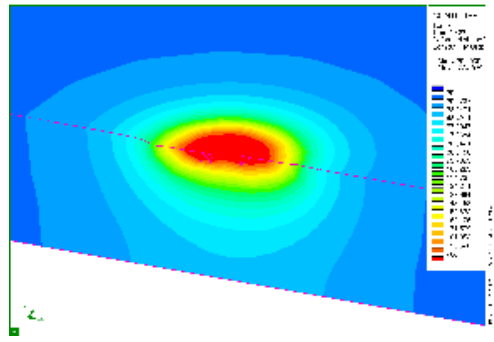

(c) $t=0.436 \mathrm{~s}$

Figure 7: Évolution du champ de température.

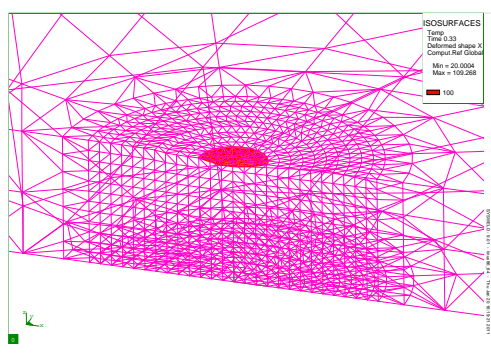

(a) $t=0.33 \mathrm{~s}$

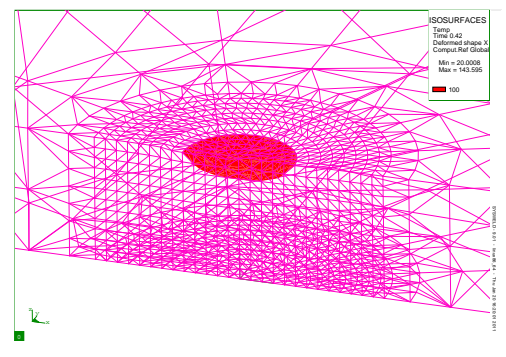

(b) $t=0.42 \mathrm{~s}$

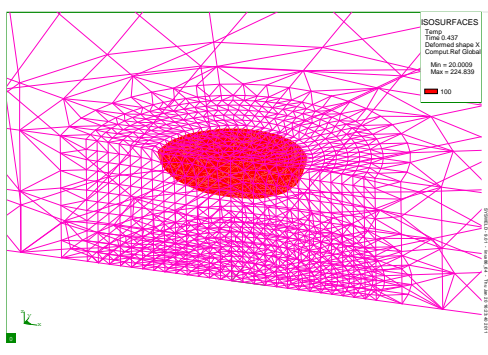

(c) $t=0.437 \mathrm{~s}$

Figure 8: Évolution de l'isotherme associée à l'interface fluide/solide.

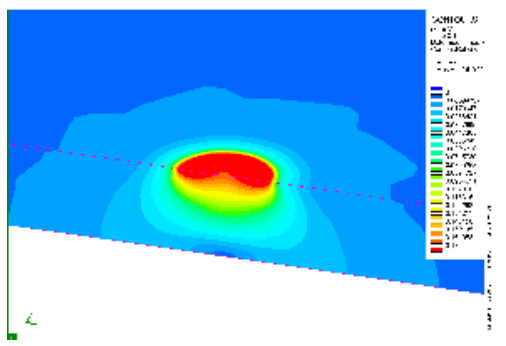

(a) $t=0.01 \mathrm{~s}$

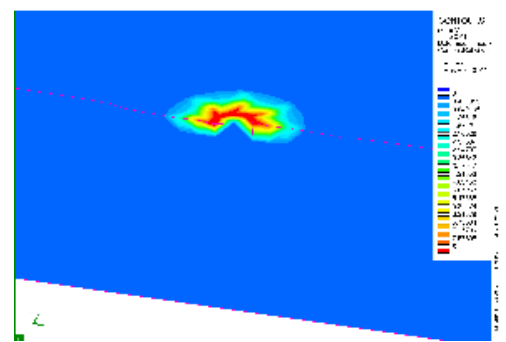

(b) $t=0.41 \mathrm{~s}$

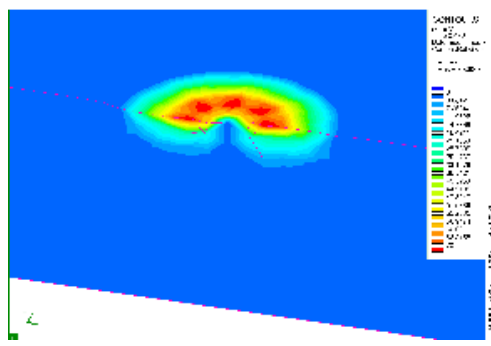

(c) $t=0.436 \mathrm{~s}$

Figure 9: Évolution de la norme de la vitesse matérielle.

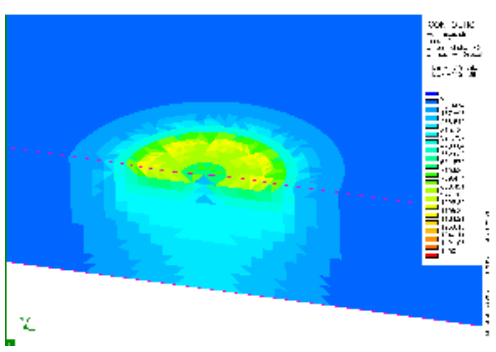

(a) $t=0.01 \mathrm{~s}$

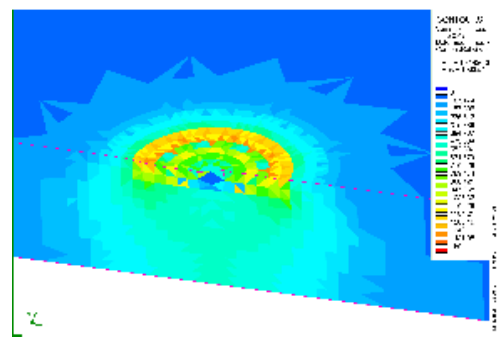

(b) $t=0.42 \mathrm{~s}$

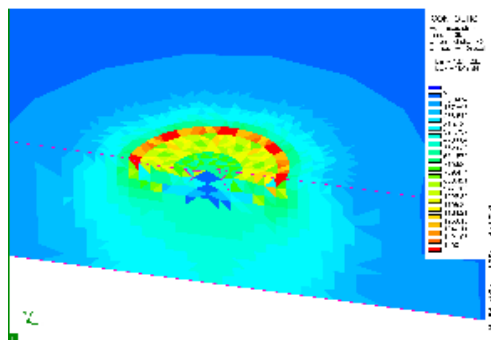

(c) $t=0.436 \mathrm{~s}$

Figure 10: Évolution de la contrainte équivalente de Mises.

\section{Conclusions}

Le Friction Stir Spot Welding est un procédé récent permettant de réaliser un joint soudé « ponctuel» à l'état solide et sans apport de matière, il s'avère particulièrement intéressant pour réaliser l'assemblage de métaux non-ferreux tels 
que les alliages d'aluminium ou de cuivre, réputés peu soudables avec les procédés classiques. Du point de vue de la modélisation, on peut identifier deux phases lors du procédé, la première au voisinage de l'outil subissant de forts taux de déformation atteint un état pâteux, le reste de la structure se déformant à l'état solide.

On a construit dans ce travail un couplage fluide/solide appliqué à la simulation numérique des procédés de soudage dans lesquels deux phases peuvent être identifiées, le Friction Stir Spot Welding représentant une première étape avant les procédés de soudage à l'arc. Ce type d'approche permet de décrire correctement l'état de la matière dans chaque partie de la structure, et de tirer profit des traitements numériques associés usuellement à chaque type de comportement. De façon plus générale, cette vision permet de concilier les approches usuelles en simulation numérique du soudage qui se focalisaient soit sur la modélisation du procédé lui-même (le bain fondu dans le cas des procédés de soudage par fusion) soit sur les conséquences induites, c'est-à-dire les contraintes et distorsions résiduelles.

L'élément $\mathrm{P} 1+/ \mathrm{P} 1$ sert de base au développement du couplage. Cet élément linéaire enrichi permet de traiter au travers d'une formulation mixte une liaison interne de type incompressibilité. Cet élément a été développé avec une formulation température/vitesse/pression en mécanique des fluides (pour un écoulement laminaire incompressible transitoire $[3,4]$ ) et en mécanique des solides dans le cadre des grandes transformations. Ces deux éléments sont couplés de façon naturelle de part leurs formulations, et intègrent une nouvelle option créée spécialement dans le code SYSWELD ${ }^{\circledR}[9]$. La transition fluide/solide est effectuée au moyen d'un test explicite sur une température moyenne par élément, l'interface passant par les faces des éléments du maillage. Le couplage est associé à une approche euléro-lagrangienne, permettant de suivre la matière dans la partie solide mais pas dans la partie fluide. La procédure d'actualisation de la géométrie est effectuée à convergence.

Enfin, une première simulation simplifiée du Friction Stir Spot Welding a permis d'illustrer le comportement du couplage, notamment l'apparition puis la progression d'une phase fluide sous l'outil. Les vitesses atteintes dans la partie fluide lors de la simulation (Figure 9(c)) montrent que le modèle permet de simuler plusieurs tours de l'outil dans la matière. Ce modèle sera étendu dans le futur (i) pour le Friction Stir Spot Welding, il apparaît nécessaire dans un premier temps d'améliorer la description de l'interaction outil/matière avec la prise en compte du contact et d'une loi de frottement, et (ii) pour la simulation des procédés de soudage à l'arc, en introduisant notamment la prise en compte de tensions de surface, permettant de simuler l'effet Marangoni [11].

\section{Références}

[1] Arnold, D.N., Brezzi, F., Fortin, M. 1984 A stable finite element for the Stokes equations. Calcolo. 21 337-344

[2] Bellet, M., Fachinotti, V.D. 2004 ALE method for solidification modelling. Computer Methods in Applied Mechanics and Engineering. 193 4355-4381

[3] Feulvarch, E., Boitout, F., Bergheau, J.M. 2007 Friction Stir Welding : modélisation de l'écoulement de la matière pendant la phase de soudage. European Journal of Computational Mechanics, Numéro spécial «Mécanique des fluides ». 16 865-887

[4] Heuzé, T., Leblond, J.B., Bergheau, J.M., Feulvarch, E. 2010 A finite element for laminar flow of incompressible fluids with inertia effects and thermomechanical coupling. European Journal of Computational Mechanics. 19 293-304

[5] Heuzé, T. 2011 Modélisation des couplages fluide/solide dans les procédés d'assemblage à haute température. Thèse de doctorat de l'Université Pierre et Marie Curie - Paris 6.

[6] Jaouen, O. 1998 Modélisation tridimensionnelle par éléments finis pour l'analyse thermo-mécanique du refroidissement des pièces coulées. Thèse de doctorat de l'École des Mines de Paris.

[7] Pan, T.Y. 2007 Friction Stir Spot Welding (FSSW) - A literature review. SAE Technical Series : 2007-01-1702.

[8] Sakano, R., Murakami, K., Yamashita, K., Hyoe, T., Fujimoto, M., Inuzuka, M., Nagao, U., Kashiki, H. 2001 Development of spot FSW robot system for automobile body members. Third International Symposium of Friction Stir Welding. Kobe, Japan.

[9] SYSWELD ${ }^{\circledR} 2007$ User's Manual. ESI Group.

[10] Thomas, W.M., Nicholas, E.D., Smith, S.D. 1991 Proceedings of the TMS 2001 Aluminum Automotive and Joining Sessions, TMS. S.K. Das, J.G. Kaufman, T.J. Lienert (Eds.), Aluminum 2001.

[11] Tsai, M.C., Sindo, K. 1989 Marangoni convection in weld pools with a free surface. International Journal for Numerical Methods in Fluids. 9 1503-1516 